\title{
Higher Prevalence of Diabetes in Pontine Infarction than in Other Posterior Circulation Strokes
}

\author{
Jinmao Zhu, ${ }^{1}$ Youfu Li, ${ }^{1}$ Yanxia Wang, ${ }^{1}$ Shuanggen Zhu $\mathbb{D}^{2}$, and Yongjun Jiang $\mathbb{D}^{1}$ \\ ${ }^{1}$ Department of Neurology, The Second Affiliated Hospital of Guangzhou Medical University, 250 Changgang East Road, \\ Guangzhou 510260, China \\ ${ }^{2}$ Department of Neurology, People's Hospital of Longhua, Shenzhen 518109, China
}

Correspondence should be addressed to Shuanggen Zhu; 45353502@qq.com and Yongjun Jiang; jiangyjnju@gmail.com

Received 24 July 2021; Revised 10 December 2021; Accepted 8 January 2022; Published 27 January 2022

Academic Editor: Carol Forsblom

Copyright (c) 2022 Jinmao Zhu et al. This is an open access article distributed under the Creative Commons Attribution License, which permits unrestricted use, distribution, and reproduction in any medium, provided the original work is properly cited.

Background. Pontine infarction is the major subtype of posterior circulation stroke, and diabetes is more common in pontine infarction patients than in anterior circulation stroke patients. Whether the prevalence of diabetes remains homogenous within the posterior circulation stroke population is unclear. The present study is aimed at investigating the prevalence of diabetes in pontine infarction and comparing it to other subtypes of posterior circulation stroke. Methods. We conducted a multicenter case-control study. Patients with posterior circulation stroke were screened. The subjects were divided into pontine infarction and nonpontine infarction groups. Results. From November 1, 2018, to February 28, 2021, a total of 6145 stroke patients were screened and 2627 patients had posterior circulation strokes. After excluding cardioembolic stroke, as well as its other determined and undetermined causes, 1549 patients with 754 pontine infarctions were included in the analysis. The prevalence of diabetes in the pontine infarction group was higher than that in the nonpontine infarction group $(42.7 \%$ vs. $31.4 \%, P<0.05$ ). After adjusting for confounding factors, diabetes was an independent risk factor for pontine infarction (OR $1.63,95 \% \mathrm{CI}$ $1.27-2.09, P<0.05)$. For small vessel occlusion, diabetes was also more common in the pontine infarction group (43.2\% vs. $30.0 \%, P<0.05)$. Multivariate analysis also showed that diabetes was an independent risk factor for pontine infarction (OR $1.80,95 \%$ CI 1.32-2.46, $P<0.05)$. Conclusion. In comparison with the nonpontine infarction subtype of posterior circulation stroke, patients with pontine infarction had a higher prevalence of diabetes, and diabetes was an independent risk factor for pontine infarction.

\section{Introduction}

As the population ages, the prevalence of diabetes has increased dramatically in recent decades. It has increased from less than $1 \%$ to $11.6 \%$ in China, which has the largest diabetic population [1]. Diabetes is a fundamental issue for patients with atherosclerosis, and it has been recognized as a well-established risk factor for ischemic stroke [2]. Many studies have demonstrated that diabetes is significantly associated with stroke location $[3,4]$ because there is more diabetes in posterior circulation stroke than in anterior circulation stroke [3]. The possible mechanism was that the vertebrobasilar artery was more sensitive to hyperglycemia. Takahashi et al. found that diabetic patients had more plaques in the vertebrobasilar artery. Moreover, the verteb- robasilar artery plaques in diabetic patients were prone to rupture [5]. Thus, more attention should be given to diabetes in posterior circulation stroke.

Within the posterior circulation, the anatomy and physiology of the artery were heterogeneous among the different segments. Thus, the prevalence of diabetes may vary among the different subtypes of posterior circulation stroke. Pontine infarction is the most common subtype and accounts for more than $40 \%$ of posterior circulation strokes [6]. The prevalence of diabetes in pontine infarction varies from $28.5 \%$ to $59.4 \%[6,7]$. In comparison to nonpontine infarction, diabetes was more common in pontine infarction [8]. Whether the posterior circulation or diabetes has a unique role in pontine infarction remains unclear. Therefore, it is necessary to compare the prevalence of diabetes in pontine 
infarction with its prevalence in other subtypes of posterior circulation stroke, a comparison that has been lacking in previous reports.

In the present study, we aimed to investigate the prevalence of diabetes in pontine infarction and to compare it with that in nonpontine subtypes of posterior circulation stroke.

\section{Methods}

2.1. Study Design and Setting. This was a multicenter casecontrol study, which was conducted from November 1, 2018 , to February 28, 2021. The study was approved by the Ethics Committee of the Second Affiliated Hospital of Guangzhou Medical University and Longhua Hospital. Informed consent was signed by the patient or the patient's authorized person.

2.2. Participants. Patients at the Second Affiliated Hospital of Guangzhou Medical University and Longhua Hospital were consecutively screened and enrolled if they met the following inclusion criteria: (1) older than 18 years old; (2) symptoms of acute stroke; (3) new infarction identified by diffusion-weighted imaging (DWI) within 7 days of stroke onset; (4) lesion located in the territory of posterior circulation; (5) intracranial and extracranial cerebral vessels evaluated by ultrasound, CTA, MRA, or DSA; and (6) infarction caused by atherosclerosis and arteriosclerosis. Patients were excluded if they met any of the following criteria: (1) missing clinical or imaging information; (2) nonatherosclerotic stroke; (3) brain tumor; (4) intracranial metastatic tumor; (5) intracerebral hemorrhage; or (6) traumatic brain injury.

2.3. Variables. A current smoker is defined as someone who smokes on some days or every day. An ex-smoker is defined as someone who has smoked $\geq 100$ cigarettes in their lifetime but has not smoked in the past 28 days [9].

A former drinker is defined as someone who once drank but who did not drink during the past year; a current light drinker is defined as someone who in the past year has had 1-12 drinks but has 3 drinks or fewer per week on average; and a current heavy drinker is defined as someone who has had at least 3 drinks per week on average over the past year [10].

Hypertension is diagnosed when someone's systolic blood pressure in the office or clinic is $\geq 140 \mathrm{mmHg}$ and/or their diastolic blood pressure is $\geq 90 \mathrm{mmHg}$ following repeated examinations [11].

Diabetes is diagnosed when someone has the classical symptoms of hyperglycemia or hyperglycemic crisis with $\mathrm{HbA} 1 \mathrm{c} \geq 6.5 \%$ (the test should be performed in a laboratory using a method that is NGSP certified and standardized to the DCCT assay), fasting plasma glucose $\geq 7.0 \mathrm{mmol} / \mathrm{L}$ (fasting is defined as no caloric intake for at least $8 \mathrm{~h}$ ), $2 \mathrm{~h}$ plasma glucose $\geq 111 \mathrm{mmol} / \mathrm{L}$ during a $75 \mathrm{~g}$ oral glucose tolerance test (OGTT), or a random plasma glucose $\geq 11.1 \mathrm{mmol} / \mathrm{L}$. Poorly controlled diabetes was defined as $\mathrm{HbAlc} \geq 7 \%$.
Someone with an HbAlc level of less than $7 \%$ is considered well controlled [12].

The classification of stroke subtypes using Trials of Org 10172 in Acute Stroke Treatment (TOAST) is as follows: (1) large-artery atherosclerosis, (2) cardioembolism, (3) small-vessel occlusion (SVO), (4) stroke of other determined etiology, and (5) stroke of undetermined etiology [13]. Stroke caused by large artery atherosclerosis and SVO were included in the analysis.

Information about medication usage was collected before stroke onset.

Pontine infarction was defined as an acute infarction located in the pons regardless of whether there were infarctions in other areas. Nonpontine infarction was defined as an acute infarction located in the extrapontine area.

2.4. Statistics. Differences in the continuous variables were compared using the test of homogeneity of variance. Student's $t$-test was used when the normality assumption was met; otherwise, the equivalent nonparametric test was used. Differences in the categorical variables were compared using Pearson's chi-square test with post hoc analysis. Missing data points and risk factor variables recorded as unknown did not exceed 3\% for any single variable. A univariate binary logistic regression analysis was performed to determine the effects of the independent variables. Individual variables with a $P$ value $<0.1$ in the univariate analysis were used in the multivariable regression analysis, and the results were expressed as odds ratios (ORs) and 95\% confidence intervals (CIs).

\section{Results}

3.1. Participants. As shown in Figure 1, a total of 6145 stroke patients were screened. Posterior circulation strokes were found in 2627 patients with a total of 890 pontine infarctions. SVO was the leading cause of pontine infarction (65.2\%, 580/890), while cardioembolism was the leading cause of nonpontine infarction $(52.1 \%, 905 / 1737)$. Considering the limited effect of diabetes on cardioembolic stroke, as well as its other determined and undetermined causes, these cases were excluded from the analysis. Thus, 1549 subjects with 754 pontine infarctions and 795 nonpontine infarctions were included.

3.2. Association of Diabetes with Pontine Infarction. For the patients with pontine infarction, $42.7 \%$ (322/754) had diabetes, which was higher than the percentage of diabetic patients with nonpontine infarction (31.4\%, 250/795). Most of the patients with diabetes were diagnosed previously. The baseline characteristics are shown in Table 1. Univariate analysis showed that hypertension, TC, LDL, diabetes, and fasting plasma glucose were significantly different between pontine and nonpontine infarction patients (Table 2, $P<$ 0.05). Multivariate analysis showed that diabetes was an independent risk factor for pontine infarction (OR 1.63, 95\% CI 1.27-2.09, $P<0.05$, Table 2).

3.3. Association of Diabetes with SVO-Induced Pontine Infarction. A total of 580 patients had pontine infarction 


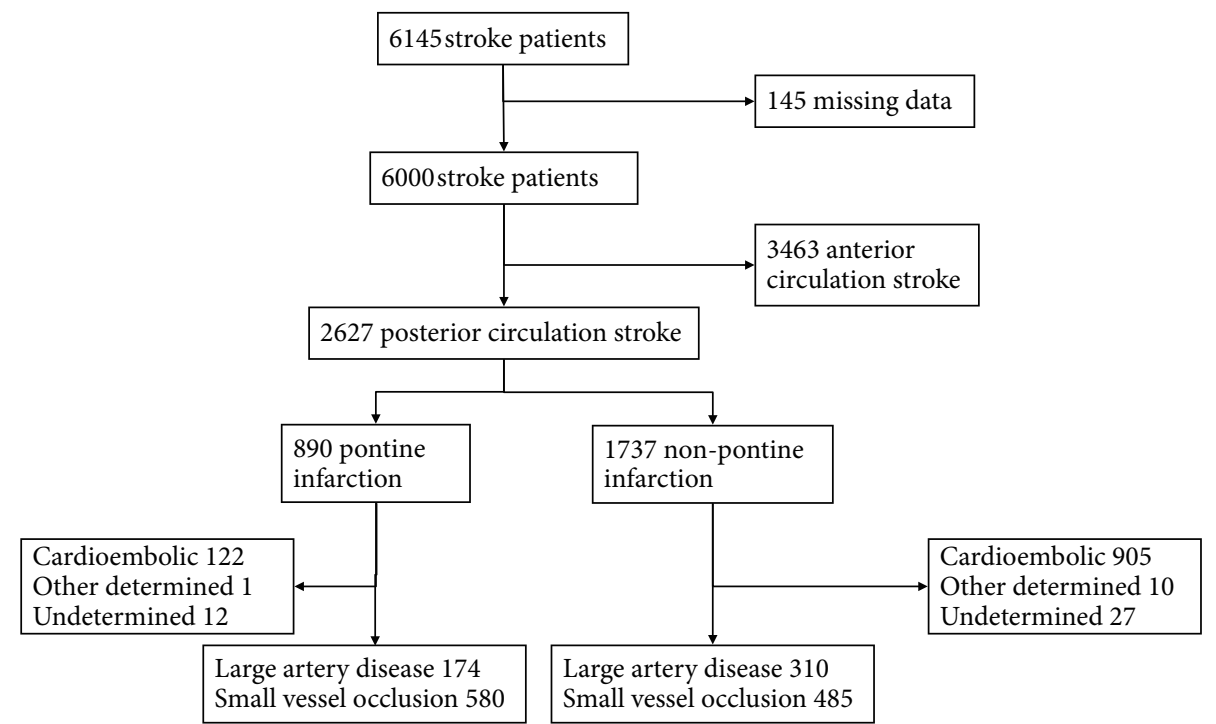

Figure 1: Flow diagram.

TABle 1: Pontine infarction vs. nonpontine infarction.

\begin{tabular}{|c|c|c|c|}
\hline & Pontine infarction & Nonpontine infarction & $P$ \\
\hline$N$ & 754 & 795 & \\
\hline Male (\%) & $475(63.0 \%)$ & $527(66.2 \%)$ & 0.184 \\
\hline Age (y) & $66.97 \pm 11.95$ & $66.03 \pm 12.15$ & 0.118 \\
\hline Smoking (\%) & $205(27.2 \%)$ & $238(29.9 \%)$ & 0.432 \\
\hline Ex-smoker (\%) & $47(22.9 \%)$ & $50(21.0 \%)$ & \\
\hline Current smoker (\%) & $158(77.1 \%)$ & $188(79.0 \%)$ & \\
\hline Drinking & $82(10.9 \%)$ & $95(11.9 \%)$ & 0.154 \\
\hline Former drinker (\%) & $26(31.7 \%)$ & $15(15.8 \%)$ & \\
\hline Current heavy drinker (\%) & $52(63.4 \%)$ & $69(72.6 \%)$ & \\
\hline Current light drinker (\%) & $4(4.9 \%)$ & $11(11.5 \%)$ & \\
\hline Hypertension (\%) & $584(77.4 \%)$ & $582(73.2 \%)$ & 0.051 \\
\hline \multicolumn{4}{|l|}{ Hyperlipidemia } \\
\hline $\mathrm{TC}(\mathrm{mM})$ & $4.79 \pm 1.26$ & $4.61 \pm 1.19$ & $<0.01$ \\
\hline $\mathrm{LDL}(\mathrm{mM})$ & $3.18 \pm 1.08$ & $3.03 \pm 1.00$ & $<0.01$ \\
\hline HDL (mM) & $1.05 \pm 0.53$ & $1.04 \pm 0.25$ & 0.676 \\
\hline $\mathrm{TG}(\mathrm{mM})$ & $1.81 \pm 1.49$ & $1.71 \pm 1.14$ & 0.142 \\
\hline Uric acid $(\mu \mathrm{M})$ & $353.77 \pm 111.25$ & $361.10 \pm 111.38$ & 0.201 \\
\hline Previous stroke or TIA (\%) & $146(19.4 \%)$ & $176(22.1 \%)$ & 0.183 \\
\hline \multicolumn{4}{|l|}{ Medicine } \\
\hline Antiplatelet (\%) & $205(27.2 \%)$ & $186(23.4 \%)$ & 0.080 \\
\hline Statin $(\%)$ & $55(7.3 \%)$ & $70(8.8 \%)$ & 0.283 \\
\hline Antihypertensive agent (\%) & $335(44.4 \%)$ & $349(43.9 \%)$ & 0.178 \\
\hline Diabetes (\%) & $322(42.7 \%)$ & $250(31.4 \%)$ & $<0.01$ \\
\hline New diagnosis (\%) & $55(17.1 \%)$ & $50(20.0 \%)$ & \\
\hline Previously diagnosed & $267(82.9 \%)$ & $200(80.0 \%)$ & \\
\hline No treatment (\%) & $23(8.6 \%)$ & $18(9.0 \%)$ & \\
\hline Poorly controlled (\%) & $208(77.9 \%)$ & $136(68.0 \%)$ & \\
\hline Well-controlled (\%) & $37(13.8 \%)$ & $46(23.0 \%)$ & \\
\hline Fasting plasma glucose (mM) & $6.46 \pm 2.93$ & $6.11 \pm 2.60$ & 0.022 \\
\hline
\end{tabular}

TC: total cholesterol; LDL: low-density lipoprotein; HDL: high-density lipoprotein; TG: triglyceride; TIA: transient ischemic attack. 
TABLE 2: Multivariate logistic regression of pontine infarction.

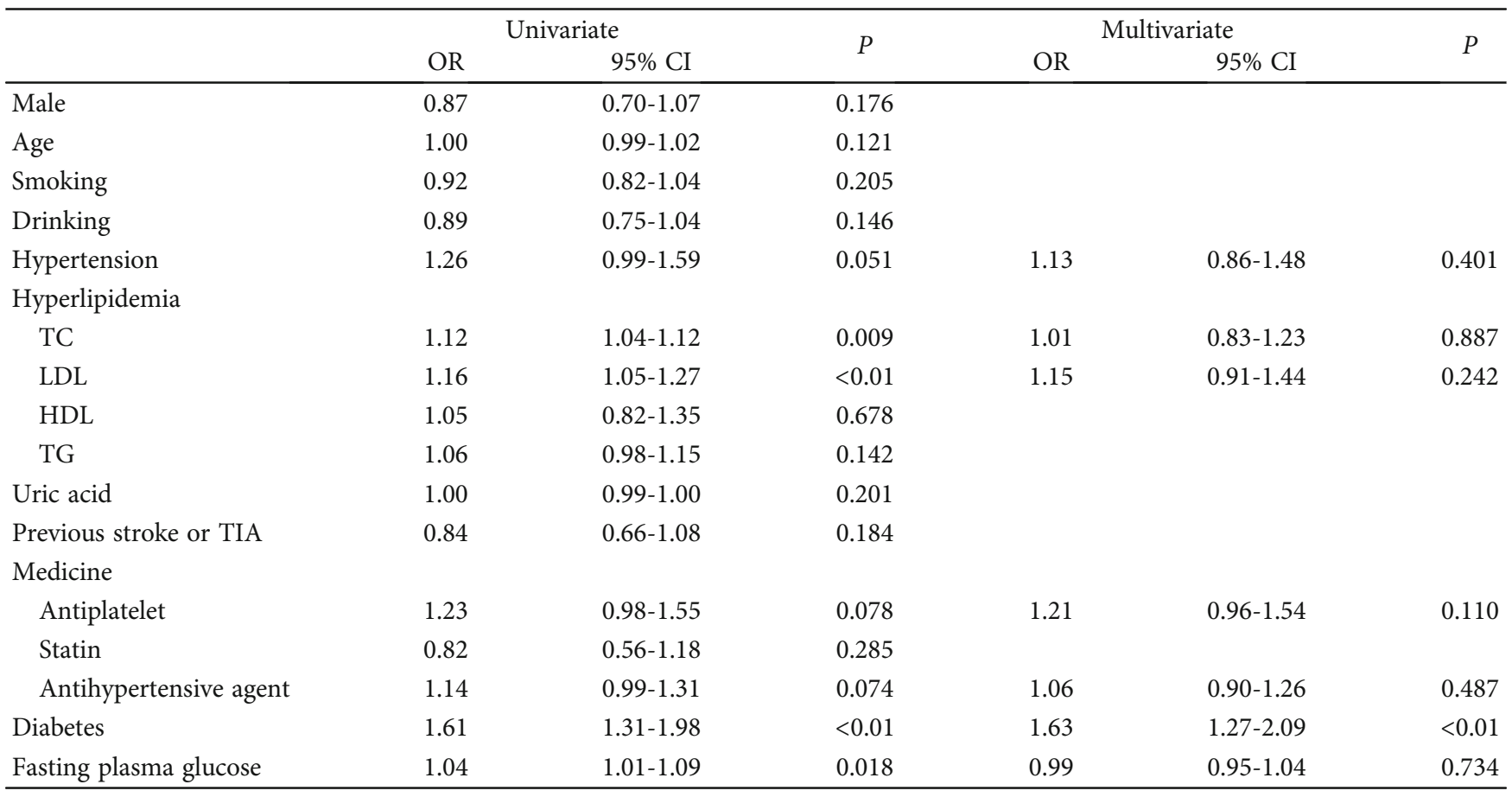

TC: total cholesterol; LDL: low-density lipoprotein; HDL: high-density lipoprotein; TG: triglyceride; TIA: transient ischemic attack.

caused by SVO, and there was a higher prevalence of diabetes in these patients than in patients with nonpontine infarction ( $43.2 \%$ vs. $30.0 \%, P<0.05$, Table 3 ). Univariate analysis showed that age, hypertension, LDL, and diabetes were significantly different between pontine and nonpontine infarction patients (Table 4). Multivariate analysis showed that diabetes was an independent risk factor for pontine infarction in SVO (OR 1.80, 95\% CI 1.32-2.46, $P<0.05$, Table 4).

\section{Discussion}

In the present study, $42.7 \%$ of patients with pontine infarction had diabetes, which was higher than that of patients with nonpontine posterior circulation stroke. Diabetes was an independent risk factor for pontine infarction even after adjusting for confounding factors. Our study demonstrated the unique role of diabetes in pontine infarction. More attention should be given to diabetes in pontine infarction.

Our data showed that the prevalence of diabetes in pontine infarction patients was $42.7 \%$. The patients in our study were Chinese. The diabetes prevalence in pontine infarction varied from $28.5 \%$ to $59.4 \%$ in previous studies, which recruited both Asians and non-Asians (Table 5). The mean prevalence of diabetes derived from the historical data was $39.0 \%$, which was very close to our prevalence (Table 5). Thus, the prevalence of diabetes in pontine infarction was not affected by ethnic origins. This prevalence was much higher than that of nonpontine stroke. The prevalence of diabetes in the HERMES meta-analysis was $16.2 \%$ [14]. In the ECASS III trial, $15.7 \%$ of subjects had diabetes [15]. In the CHANCE trial, which mainly included Chinese stroke patients, $21.1 \%$ had diabetes [16]. In a head-tohead study, Nakase et al. found that the rate of diabetes was higher in patients with pontine infarction than in those without pontine infarction. However, nonpontine infarction includes both anterior and posterior circulation strokes [17]. Considering the higher rate of diabetes in posterior circulation stroke [3], one cannot exclude the possibility that it was because pontine infarction is a type of posterior circulation stroke. In the present study, $31.4 \%$ of patients with nonpontine infarction had diabetes, which was less than that of pontine infarction patients. Even after adjusting for confounding factors, diabetes was an independent risk factor for pontine infarction.

We found that SVO was the most common mechanism of pontine infarction, which was consistent with other studies [6]. Diabetes was associated more with SVO. Li et al. found that patients with pontine infarction caused by SVO were more likely to have diabetes than those with SVO-induced nonpontine infarction in the anterior circulation [18]. Thus, we performed a further subgroup analysis of patients with SVO. Among the patients with SVO, diabetes was also more common in pontine infarction than in nonpontine infarction of posterior circulation stroke. All of this evidence suggested that diabetes has a unique role in pontine infarction.

The arteries supplying the pons are the pontine arteries, which come off at right angles from the basilar artery. The mechanism underlying the greater susceptibility of the basilar artery to diabetes remains unknown [19]. Calcification in 
TABLE 3: Pontine infarction vs. nonpontine infarction with SVO.

\begin{tabular}{|c|c|c|c|}
\hline & Pontine infarction & Nonpontine infarction & $P$ \\
\hline$N$ & 580 & 486 & \\
\hline Male (\%) & $370(63.8 \%)$ & $321(66.0 \%)$ & 0.442 \\
\hline Age (y) & $67.14 \pm 11.76$ & $65.38 \pm 11.94$ & 0.018 \\
\hline Smoking (\%) & $158(27.2 \%)$ & $142(29.2 \%)$ & 0.774 \\
\hline Ex-smoker (\%) & $34(21.5 \%)$ & $30(21.2 \%)$ & \\
\hline Current smoker (\%) & $124(78.5 \%)$ & $112(78.8 \%)$ & \\
\hline Drinking & $61(10.5 \%)$ & $56(11.5 \%)$ & 0.123 \\
\hline Former drinker (\%) & $18(29.5 \%)$ & $9(16.1 \%)$ & \\
\hline Current heavy drinker (\%) & $41(67.2 \%)$ & $40(71.4 \%)$ & \\
\hline Current light drinker (\%) & $2(3.3 \%)$ & $7(12.5 \%)$ & \\
\hline Hypertension (\%) & $444(76.6 \%)$ & $343(70.6 \%)$ & 0.028 \\
\hline \multicolumn{4}{|l|}{ Hyperlipidemia } \\
\hline $\mathrm{TC}(\mathrm{mM})$ & $4.81 \pm 1.29$ & $4.66 \pm 1.20$ & 0.062 \\
\hline $\mathrm{LDL}(\mathrm{mM})$ & $3.20 \pm 1.10$ & $3.04 \pm 1.00$ & 0.018 \\
\hline $\mathrm{HDL}(\mathrm{mM})$ & $1.04 \pm 0.28$ & $1.04 \pm 0.25$ & 0.990 \\
\hline $\mathrm{TG}(\mathrm{mM})$ & $1.85 \pm 1.61$ & $1.82 \pm 1.28$ & 0.791 \\
\hline Uric acid $(\mu \mathrm{M})$ & $356.08 \pm 107.43$ & $356.89 \pm 103.66$ & 0.868 \\
\hline Previous stroke or TIA (\%) & $108(18.6 \%)$ & $102(20.9 \%)$ & 0.330 \\
\hline Coronal heart disease (\%) & $44(7.6 \%)$ & $37(7.6 \%)$ & 0.996 \\
\hline \multicolumn{4}{|l|}{ Medicine } \\
\hline Antiplatelet (\%) & $157(27.0 \%)$ & $116(23.9 \%)$ & 0.228 \\
\hline Statin $(\%)$ & $40(6.9 \%)$ & $46(9.5 \%)$ & 0.131 \\
\hline Antihypertensive agent (\%) & $247(42.6 \%)$ & $207(42.5 \%)$ & 0.232 \\
\hline Diabetes (\%) & $251(43.2 \%)$ & $146(30.0 \%)$ & $<0.01$ \\
\hline New diagnosis (\%) & $38(15.1 \%)$ & $31(21.2 \%)$ & 0.374 \\
\hline No drugs (\%) & $15(5.9 \%)$ & $9(6.1 \%)$ & \\
\hline Poorly controlled (\%) & $161(64.1 \%)$ & $79(52.0 \%)$ & \\
\hline Well-controlled (\%) & $33(13.1 \%)$ & $27(18.5 \%)$ & \\
\hline Fasting plasma glucose (mM) & $6.45 \pm 3.04$ & $6.12 \pm 2.62$ & 0.058 \\
\hline
\end{tabular}

TC: total cholesterol; LDL: low-density lipoprotein; HDL: high-density lipoprotein; TG: triglyceride; TIA: transient ischemic attack.

atherosclerotic plaques may predispose them to rupture by inducing mechanical instability. Calcification of basilar artery plaques was significantly related to diabetes [20]. The degree of calcification was positively related to the level of blood glucose [21]. An experimental study showed that relaxation of the basilar artery was negatively affected by diabetes [22], which resulted in constriction of the basilar artery to promote atherosclerosis [23]. The mechanism might be that large-conductance $\mathrm{Ca}^{2+}$-activated potassium channels in basilar artery smooth muscle cells were impaired by diabetes [24].

Most of the patients with previously diagnosed diabetes received treatment, including drug and/or nondrug treatments. The rate of treatment was higher than the average in China. However, for the majority of patients receiving treatment, their diabetes was poorly controlled. Higher $\mathrm{HbAlc}$ was related to early neurologic deterioration and the long-term poor prognosis of pontine infarction
$[25,26]$. Moreover, HbA1c could promote the progression of TIA to stroke in the posterior circulation [27]. Therefore, a tight glucose control strategy was necessary for the prevention of pontine infarction.

There were some limitations of the study. First, the diagnosis of infarction in the present study was based on DWI images. However, some patients, especially those with brainstem strokes, had DWI-negative strokes. This would lead to some bias. Second, the diagnosis of SVO was mostly made based on the infarction distribution. Some studies have used high-resolution MRI (HR-MRI) to detect branch artery disease [28]. Some of our subjects also received HR-MRI. However, not all branch arteries could be detected by HR-MRI. Finally, although our study was one of the largest in terms of the number of pontine infarction patients, more subjects and more studies are needed to investigate the relationship between diabetes and pontine infarction. 
TABLE 4: Multivariate logistic regression of pontine infarction with SVO.

\begin{tabular}{|c|c|c|c|c|c|c|}
\hline & \multicolumn{2}{|c|}{ Univariate } & \multirow{2}{*}{$P$} & \multicolumn{2}{|c|}{ Multivariate } & \multirow{2}{*}{$P$} \\
\hline & OR & $95 \% \mathrm{CI}$ & & OR & $95 \% \mathrm{CI}$ & \\
\hline Male & 0.91 & $0.70-1.17$ & 0.441 & & & \\
\hline Age & 1.01 & $1.00-1.02$ & 0.019 & 1.01 & $1.00-1.02$ & 0.052 \\
\hline Smoking & 0.95 & $0.82-1.07$ & 0.483 & & & \\
\hline Drinking & 0.89 & $0.73-1.08$ & 0.240 & & & \\
\hline Hypertension & 1.36 & $1.03-1.79$ & 0.032 & 1.24 & $0.94-1.65$ & 0.132 \\
\hline \multicolumn{7}{|l|}{ Hyperlipidemia } \\
\hline TC & 1.10 & $0.99-1.22$ & 0.058 & 0.93 & $0.74-1.16$ & 0.521 \\
\hline LDL & 1.15 & $1.02-1.30$ & 0.021 & 1.26 & $0.97-1.65$ & 0.089 \\
\hline HDL & 1.00 & $0.63-1.58$ & 0.987 & & & \\
\hline TG & 1.01 & $0.93-1.10$ & 0.789 & & & \\
\hline Uric acid & 1.00 & $0.99-1.00$ & 0.362 & & & \\
\hline Previous stroke or TIA & 0.86 & $0.64-1.16$ & 0.332 & & & \\
\hline \multicolumn{7}{|l|}{ Medicine } \\
\hline Antiplatelet & 1.18 & $0.90-1.56$ & 0.230 & & & \\
\hline Statin & 0.71 & $1.45-1.10$ & 0.131 & & & \\
\hline Antihypertensive agent & 1.15 & $0.97-1.36$ & 0.108 & & & \\
\hline Diabetes & 1.73 & $0.33-2.23$ & $<0.01$ & 1.80 & $1.32-2.46$ & $<0.01$ \\
\hline Fasting plasma glucose & 1.04 & $0.99-1.09$ & 0.062 & 0.99 & $0.94-1.04$ & 0.601 \\
\hline
\end{tabular}

TC: total cholesterol; LDL: low-density lipoprotein; HDL: high-density lipoprotein; TG: triglyceride; TIA: transient ischemic attack.

TABle 5: Previous reports of diabetes in pontine infarction.

\begin{tabular}{|c|c|c|c|c|}
\hline Year & Author & Diabetes & Pontine infarction & Prevalence (\%) \\
\hline 1994 & Toyoda [29] & 29 & 73 & 39.73 \\
\hline 2002 & Kumral [30] & 45 & 150 & 30.00 \\
\hline 2005 & Vemmos [31] & 29 & 100 & 29.00 \\
\hline 2008 & Liang [32] & 4 & 14 & 28.57 \\
\hline 2009 & Kwon [33] & 49 & 96 & 51.04 \\
\hline 2009 & Liang [34] & 4 & 17 & 23.53 \\
\hline 2010 & Aoki [35] & 25 & 51 & 49.02 \\
\hline 2010 & Klein [36] & 13 & 45 & 28.89 \\
\hline 2012 & Oh [37] & 87 & 200 & 43.50 \\
\hline 2013 & Feng [38] & 25 & 81 & 30.86 \\
\hline 2013 & $\mathrm{Ju}$ [39] & 60 & 101 & 59.40 \\
\hline 2014 & Nakase [40] & 13 & 38 & 34.20 \\
\hline 2015 & Feng [41] & 19 & 55 & 34.54 \\
\hline 2015 & $\operatorname{Lim}[42]$ & 36 & 87 & 41.38 \\
\hline 2016 & Huang [43] & 147 & 265 & 55.47 \\
\hline 2016 & Wilson [7] & 189 & 619 & 30.53 \\
\hline 2017 & Gokcal [44] & 69 & 120 & 57.50 \\
\hline 2017 & Lapa [45] & 31 & 59 & 52.54 \\
\hline 2018 & Zhou [6] & 61 & 175 & 34.86 \\
\hline 2019 & Huang [46] & 372 & 1003 & 37.09 \\
\hline \multicolumn{2}{|r|}{ Total } & 1307 & 3349 & 39.03 \\
\hline
\end{tabular}




\section{Conclusion}

In conclusion, the prevalence of diabetes was higher in pontine infarction than in the other subtypes of posterior circulation stroke, and diabetes was an independent risk factor for pontine infarction.

\section{Data Availability}

All data was available.

\section{Conflicts of Interest}

The authors declare that they have no conflicts of interest.

\section{Authors' Contributions}

Jinmao Zhu, Youfu Li, and Yanxia Wang contributed equally to this manuscript.

\section{Acknowledgments}

This study was financially supported by the National Science Foundation of China (81870933), the Guangzhou Science and Technology Project (202102010127), the Guangdong Basic and Applied Basic Research Foundation (2021A1515012351), and the Opening Lab Program of Guangzhou Medical University (0506308) to Y Jiang and High Level Project of Medicine in Longhua, Shenzhen (HLPM201907020102), and Construction Funds of Key Medical Disciplines in Longhua District, Shenzhen (MKD202007090208) to Zhu.

\section{References}

[1] X. Liu, Y. Li, L. Li et al., "Prevalence, awareness, treatment, control of type 2 diabetes mellitus and risk factors in chinese rural population: the ruraldiab study," Scientific Reports, vol. 6, no. 1, article 31426, 2016.

[2] B. Stegmayr and K. Asplund, "Diabetes as a risk factor for stroke. A population perspective," Diabetologia, vol. 38, pp. 1061-1068, 1995.

[3] G. Coban, E. Cifci, E. Yildirim, and A. M. Agildere, "Predisposing factors in posterior circulation infarcts: a vascular morphological assessment," Neuroradiology, vol. 57, pp. 483-489, 2015.

[4] S. Palacio, L. A. McClure, O. R. Benavente, C. Bazan 3rd, P. Pergola, and R. G. Hart, "Lacunar strokes in patients with diabetes mellitus: risk factors, infarct location, and prognosis: the secondary prevention of small subcortical strokes study," Stroke; A Journal of Cerebral Circulation, vol. 45, no. 9, pp. 2689-2694, 2014.

[5] Y. Takahashi, T. Yamashita, R. Morihara et al., "Different characteristics of anterior and posterior branch atheromatous diseases with or without early neurologic deterioration," Journal of stroke and Cerebrovascular Diseases: The Official Journal of National Stroke Association, vol. 26, pp. 1314-1320, 2017.

[6] L. Zhou, M. Yao, B. Peng, Y. Zhu, J. Ni, and L. Cui, "Atherosclerosis might be responsible for branch artery disease: evidence from white matter hyperintensity burden in acute isolated pontine infarction," Frontiers in Neurology, vol. 9, p. 840, 2018.

[7] L. K. Wilson, L. A. Pearce, A. Arauz et al., "Morphological classification of penetrating artery pontine infarcts and association with risk factors and prognosis: the sps 3 trial," International Journal of Stroke: Official Journal of the International Stroke Society, vol. 11, pp. 412-419, 2016.

[8] H. Ichikawa, A. Kuriki, R. Kinno, H. Katoh, M. Mukai, and M. Kawamura, "Occurrence and clinicotopographical correlates of brainstem infarction in patients with diabetes mellitus," Journal of Stroke and Cerebrovascular Diseases: The Official Journal of National Stroke Association, vol. 21, pp. 890-897, 2012.

[9] H. Ryan, A. Trosclair, and J. Gfroerer, "Adult Current Smoking: Differences in Definitions and Prevalence Estimates-NHIS and NSDUH, 2008," Journal of Environmental and Public Health, vol. 2012, Article ID 918368, 11 pages, 2012.

[10] D. Zheng, Z. Mingyue, S. Wei et al., "The incidence and risk factors of in-stent restenosis for vertebrobasilar artery stenting," World Neurosurgery, vol. 110, pp. e937-e941, 2018.

[11] T. Unger, C. Borghi, F. Charchar et al., "2020 international society of hypertension global hypertension practice guidelines," Journal of Hypertension, vol. 38, pp. 982-1004, 2020.

[12] M. Raoufi, S. Khalili, M. Mansouri, A. Mahdavi, and N. Khalili, "Well-controlled vs poorly-controlled diabetes in patients with covid-19: are there any differences in outcomes and imaging findings?," Diabetes Research and Clinical Practice, vol. 166, p. 108286, 2020.

[13] H. P. Adams Jr., B. H. Bendixen, L. J. Kappelle et al., "Classification of subtype of acute ischemic stroke. Definitions for use in a multicenter clinical trial. Toast. Trial of org 10172 in acute stroke treatment," Stroke; A Journal of Cerebral Circulation, vol. 24, pp. 35-41, 1993.

[14] M. A. Almekhlafi, M. D. Hill, Y. M. Roos et al., "Stroke laterality did not modify outcomes in the hermes meta-analysis of individual patient data of 7 trials," Stroke; A Journal of Cerebral Circulation, vol. 50, pp. 2118-2124, 2019.

[15] W. Hacke, M. Kaste, E. Bluhmki et al., "Thrombolysis with alteplase 3 to 4.5 hours after acute ischemic stroke," The New England Journal of Medicine, vol. 359, pp. 1317-1329, 2008.

[16] Y. Wang, Y. Wang, X. Zhao et al., "Clopidogrel with aspirin in acute minor stroke or transient ischemic attack," The New England Journal of Medicine, vol. 369, no. 1, pp. 11-19, 2013.

[17] T. Nakase, Y. Yamamoto, K. Ooiwa, M. Hayashi, and K. Nakajima, "Diabetes mellitus and ischemic stroke. Ischemic stroke topography for diabetes mellitus," Japanese Journal of Stroke, vol. 22, no. 2, pp. 335-342, 2000.

[18] H. Li, Y. Shu, B. Hu et al., "Characteristics of paramedian pontine arteries disease and its association with hemoglobinalc," Brain and Behavior: A Cognitive Neuroscience Perspective, vol. 8, article e00946, 2018.

[19] P. Frid, M. Drake, A. K. Giese et al., "Detailed phenotyping of posterior vs. anterior circulation ischemic stroke: a multicenter mri study," Journal of Neurology, vol. 267, no. 3, pp. 649-658, 2020.

[20] S. Pikija, J. Magdic, and T. Hojs-Fabjan, "Calcifications of vertebrobasilar arteries on ct: detailed distribution and relation to risk factors in 245 ischemic stroke patients," BioMed Research International, vol. 2013, Article ID 918970, 7 pages, 2013.

[21] E. Gokcal, E. Niftaliyev, T. Ozdemir, M. Kolukisa, and T. Asil, "The association of vertebrobasilar calcification with etiological 
subtypes, stroke recurrence and outcome in acute brainstem ischemic stroke," Neurologia i Neurochirurgia Polska, vol. 52, pp. 188-193, 2018.

[22] M. A. López-Morales, J. M. Centeno, T. Jover-Mengual et al., "Mechanisms involved in the increased sensitivity of the rabbit basilar artery to atrial natriuretic peptide in diabetes," European Journal of Pharmacology, vol. 815, pp. 64-72, 2017.

[23] T. Matsumoto, S. Yoshiyama, T. Kobayashi, and K. Kamata, "Mechanisms underlying enhanced contractile response to endothelin-1 in diabetic rat basilar artery," Peptides, vol. 25, pp. 1985-1994, 2004.

[24] Y. Wang, H. T. Zhang, X. L. Su et al., "Experimental diabetes mellitus down-regulates large-conductance ca 2+-activated $\mathrm{k}+$ channels in cerebral artery smooth muscle and alters functional conductance," Current Neurovascular Research, vol. 7, pp. 75-84, 2010.

[25] H. Li, W. Qiu, B. Hu et al., "Ischemic volumes and early neurologic deterioration in acute brainstem infarctions with hemoglobin a1c," European Neurology, vol. 70, pp. 225-232, 2013.

[26] T. Nakase, S. Yoshioka, M. Sasaki, and A. Suzuki, "Clinical evaluation of lacunar infarction and branch atheromatous disease," Journal of Stroke and Cerebrovascular Diseases: The Official Journal of National Stroke Association, vol. 22, pp. 406412, 2013.

[27] J. Wang, J. Wu, R. Liu, F. Gao, H. Hu, and X. Yin, "The $\mathrm{ABCD} 2$ score is better for stroke risk prediction after anterior circulation tia compared to posterior circulation tia," The International Journal of Neuroscience, vol. 125, no. 1, pp. 5055,2015

[28] S. H. Lee, S. C. Jung, D. W. Kang, S. U. Kwon, and J. S. Kim, "Visualization of culprit perforators in anterolateral pontine infarction: high-resolution magnetic resonance imaging study," European Neurology, vol. 78, pp. 229-233, 2017.

[29] K. Toyoda, Y. Saku, S. Ibayashi, S. Sadoshima, T. Ogasawara, and M. Fujishima, "Pontine infarction extending to the basal surface," Stroke; A Journal of Cerebral Circulation, vol. 25, pp. 2171-2178, 1994.

[30] E. Kumral, G. Bayulkem, and D. Evyapan, "Clinical spectrum of pontine infarction," Clinical-mri Correlations. Journal of Neurology, vol. 249, no. 12, pp. 1659-1670, 2002.

[31] K. N. Vemmos, K. Spengos, G. Tsivgoulis, E. Manios, V. Zis, and D. Vassilopoulos, "Aetiopathogenesis and long-term outcome of isolated pontine infarcts," Journal of Neurology, vol. 252, pp. 212-217, 2005.

[32] Z. Liang, J. Zeng, C. Zhang et al., "Longitudinal investigations on the anterograde and retrograde degeneration in the pyramidal tract following pontine infarction with diffusion tensor imaging," Cerebrovascular Diseases, vol. 25, pp. 209-216, 2008.

[33] H. M. Kwon, J. H. Kim, J. S. Lim, J. H. Park, S. H. Lee, and Y. S. Lee, "Basilar artery dolichoectasia is associated with paramedian pontine infarction," Cerebrovascular Diseases, vol. 27, pp. 114-118, 2009.

[34] Z. Liang, J. Zeng, C. Zhang et al., "Progression of pathological changes in the middle cerebellar peduncle by diffusion tensor imaging correlates with lesser motor gains after pontine infarction," Neurorehabilitation and Neural Repair, vol. 23, pp. 692698, 2009.

[35] J. Aoki, Y. Iguchi, K. Kimura, K. Shibazaki, T. Iwanaga, and K. Sakai, "Diameter of the basilar artery may be associated with neurological deterioration in acute pontine infarction," European Neurology, vol. 63, pp. 221-226, 2010.

[36] I. F. Klein, P. C. Lavallee, M. Mazighi, E. Schouman-Claeys, J. Labreuche, and P. Amarenco, "Basilar artery atherosclerotic plaques in paramedian and lacunar pontine infarctions: a high-resolution mri study," Stroke; A Journal of Cerebral Circulation, vol. 41, pp. 1405-1409, 2010.

[37] S. Oh, O. Y. Bang, C. S. Chung, K. H. Lee, W. H. Chang, and G. M. Kim, "Topographic location of acute pontine infarction is associated with the development of progressive motor deficits," Stroke; A Journal of Cerebral Circulation, vol. 43, pp. 708-713, 2012.

[38] C. Feng, Y. Xu, X. Bai et al., "Basilar artery atherosclerosis and hypertensive small vessel disease in isolated pontine infarctions: a study based on high-resolution mri," European Neurology, vol. 70, pp. 16-21, 2013.

[39] Y. Ju, M. Hussain, K. Asmaro et al., "Clinical and imaging characteristics of isolated pontine infarcts: a one-year followup study," Neurological Research, vol. 35, pp. 498-504, 2013.

[40] T. Nakase, M. Sasaki, Y. Ikeda, and A. Suzuki, "Progressing small vessel pontine infarction includes different etiologies," Annals of Clinical and Translational Neurology, vol. 1, pp. 75-79, 2014.

[41] C. Feng, T. Hua, Y. Xu, X. Y. Liu, and J. Huang, “Arterial remodeling of basilar atherosclerosis in isolated pontine infarction," Neurological Sciences: Official Journal of the Italian Neurological Society and of the Italian Society of Clinical Neurophysiology, vol. 36, pp. 547-551, 2015.

[42] S. H. Lim, H. Choi, H. T. Kim et al., "Basilar plaque on highresolution mri predicts progressive motor deficits after pontine infarction," Atherosclerosis, vol. 240, pp. 278-283, 2015.

[43] R. Huang, X. Zhang, W. Chen, J. Lin, Z. Chai, and X. Yi, "Stroke subtypes and topographic locations associated with neurological deterioration in acute isolated pontine infarction," Journal of Stroke and Cerebrovascular Diseases: The Official Journal of National Stroke Association, vol. 25, pp. $206-$ 213, 2016.

[44] E. Gokcal, E. Niftaliyev, G. Baran, C. Deniz, and T. Asil, "Progressive deficit in isolated pontine infarction: the association with etiological subtype, lesion topography and outcome," Acta Neurologica Belgica, vol. 117, pp. 649-654, 2017.

[45] S. Lapa, S. Luger, W. Pfeilschifter, C. Henke, M. Wagner, and C. Foerch, "Predictors of dysphagia in acute pontine infarction," Stroke; A Journal of Cerebral Circulation, vol. 48, pp. 1397-1399, 2017.

[46] J. Huang, Z. Qiu, P. Zhou et al., "Topographic location of unisolated pontine infarction," BMC Neurology, vol. 19, p. 186, 2019. 